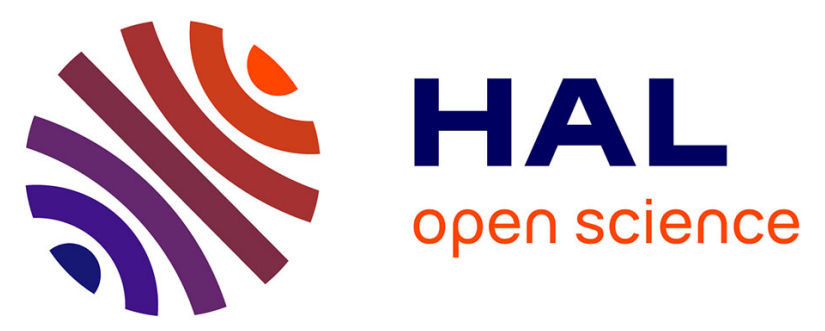

\title{
Mechanobiological model to study the influence of screw design and surface treatment on osseointegration
}

Nicolas Rousseau, Patrick Chabrand, Arnaud Destainville, Olivier Richart, Jean-Louis Milan

\section{- To cite this version:}

Nicolas Rousseau, Patrick Chabrand, Arnaud Destainville, Olivier Richart, Jean-Louis Milan. Mechanobiological model to study the influence of screw design and surface treatment on osseointegration. Computer Methods in Biomechanics and Biomedical Engineering, 2021, pp.1-17. 10.1080/10255842.2021.1950144 . hal-03467261

\section{HAL Id: hal-03467261 https://hal.science/hal-03467261}

Submitted on 6 Dec 2021

HAL is a multi-disciplinary open access archive for the deposit and dissemination of scientific research documents, whether they are published or not. The documents may come from teaching and research institutions in France or abroad, or from public or private research centers.
L'archive ouverte pluridisciplinaire HAL, est destinée au dépôt et à la diffusion de documents scientifiques de niveau recherche, publiés ou non, émanant des établissements d'enseignement et de recherche français ou étrangers, des laboratoires publics ou privés.

\section{(ㅇ)(1) $\$$}

Distributed under a Creative Commons Attribution - NonCommercial - NoDerivatives| 4.0 


\title{
Mechanobiological model to study the influence of screw design and surface treatment on osseointegration
}

\author{
Nicolas Rousseau $^{\mathrm{a}, \mathrm{b}}$, Patrick Chabrand ${ }^{\mathrm{a}}$, Arnaud Destainville ${ }^{\mathrm{c}}$, Olivier Richart ${ }^{\mathrm{b}}$ and \\ Jean-Louis Milan ${ }^{\mathrm{a}}$ \\ ${ }^{a}$ Aix Marseille Univ, CNRS, ISM, Marseille, France; belenium Medical, La Rochelle, France; \\ ${ }^{c}$ Abys Medical, La Rochelle, France
}

ARTICLE HISTORY

Compiled April 23, 2021

\begin{abstract}
This study aims at suggesting a new approach to peri-implant healing models, providing a set of taxis-diffusion-reaction equations under the combined influence of mechanical and biochemical factors. Early events of osseointegration were simulated for titanium screw implants inserted into a pre-drilled trabecular bone environment, up to twelve weeks of peri-implant bone healing. Simulations showed the ability of the model to reproduce biological events occurring at the implant interface through osteogenesis. Implants with shallow healing chamber showed higher proportions of lamellar bone, enhanced by the increase of mechanical stimulation. Osteoconduction was observed through the surface treatment model and similar bone healing patterns compared to in vivo studies.
\end{abstract}

\section{KEYWORDS}

Osseointegration; Mechanobiological model; Implant design; Surface treatment; Finite element simulation

\section{Introduction}

Screw-shaped titanium implants are commonly used for the treatment of many pathologies or traumas, from the dental field to the orthopedic one. To improve implants osseointegration in the long term, researchers and manufacturers goal focus on the constant provision of new designs with innovative geometris and surface treatments obtained through mechanical or chemical process. Osseointegration is a complex clinical state where direct and functional contact is established between an implant and bone, involving a sucession of biological events (Brånemark et al. 1969; Schroeder, Pohler, and Sutter 1976). During the surgical procedure, a bone cavity is drilled to place the implant and is filled with blood, due to vessels damage. Proteins contained in blood are adsorbed at the surface of the implant (Mavrogenis et al. 2009). This phenomenon triggers the adhesion and activation of red blood cells, platelets and inflammatory cells to secrete cytokines, growth and differentiation factors, resulting in the formation of a blood clot that acts as a scaffold for the migration of mesenchymal stem cells (MSC) and osteoblasts towards the implant. These cells, under the influence

CONTACT Nicolas Rousseau, Selenium Medical, 9049 rue de Québec, CS80875, 17043 La Rochelle, France. Tel: +335464440 82; Fax: +335463162 71; Email: nrousseau@selenium-medical.com 
of growth and differentiation factors, deposit a new and condensed collagenous matrix that forms woven bone, generating a continuity at the bone-implant interface (Meyer et al. 2004). This formation occurs both from the host bone cavity towards the implant surface and inversely : we respectively talk about distance osteogenesis (osteoinduction) and contact osteogenesis (osteoconduction). Then, woven bone is progressively remodeled into lamellar bone, showing higher degrees of mineralization and providing a stronger fixation of the implant. Such remodeling is mechanically controlled through a process called mechanotransduction, in which a physical stimulus is transformed in a biological response by osteogenic cells in order to adapt bone quality and architecture to the environment stress (Martino et al. 2018). Furthermore, implants geometry are known to play a crucial role in osseointegration, whether it is through the achievement of bone anchoring (Shiffler et al. 2016) or the establishment of a biological interface with bone (Lemons 2000). It is accepted by the scientific literature that implants surface modifications, in terms of chemical composition and topology, have an impact on their rate of osseointegration. Indeed, in vitro and in vivo studies together showed that surface treatments introducing features at micrometric or nanometric scale positively affects mesenchymal stem cells and osteoblasts, promoting a stronger adhesion to the surface, shortening their proliferation phase and enhancing their maturation to secrete more rapidly bony extracellular matrix (Schwartz et al. 2008; Szmukler-Moncler et al. 2004; Park et al. 2009; Gittens et al. 2013). Among the wide variety of tests that can be conducted in order to obtain a more comprehensive analysis of osseointegration mechanisms, in silico studies bring the advantage to quickly and cost-effectively assess hypothesis. Furthermore, although in vitro and in vivo studies give a practical approach to research on osseointegration, ethics and costs related to the duration and complexity of bone regeneration and remodeling act as a major drag to evaluate the effect of implant design and surface treatment. As a consequence during the last decades, computational models have been developed to characterize, exhaustively or only partially, mecanisms associated to these phenomena. Among these models, diffusion-reaction models have been widely used to model cellular activity such as cell migration, proliferation and differentiation. Many attempts have been conducted in the scientific literature to model peri-implant bone healing. Ambard et al. (Ambard and Swider 2006; Ambard, Guérin, and Swider 2009) emphasized the role of bone cell migration and growth factors in bone regeneration. Andreykiv et al. through a model to study the effect of a porous surface on periimplant bone cells differentiation, brought the influence of mechanical stress on cell proliferation and differentiation (Andreykiv, van Keulen, and Prendergast 2008). This model predicted cell differentiated phenotype through a criterion used by Prendergast et al. in a fracture healing computational model. The introduction of cell-implant interactions has been later covered by the work of Moreo et al. and Amor et al. who, in addition to the implementation of adsorbed proteins and platelets attachment to the implant, modified boundary conditions or cell proliferation and differentiation parameters at the periphery of the implant to model the impact of implant microtopography (Moreo, García-Aznar, and Doblaré 2009; Amor et al. 2011). These studies manage to accurately reproduce bone healing patterns in a screw healing chamber, but do not take into account both the impact of biochemical factors and mechanics on cells proliferation, differentiation, and extra-cellular matrix secretion. The objective of this study is to suggest a novel mechanobiological model to study the effect of both implant design and surface treatment on peri-implant bone healing. In particular, this model takes into account the influence of biological factors and mechanical stress in the proliferation and differentiation of bone cells, as well as in the extracellular matrix 
formation.

\section{Materials and method}

The mathematical model designed in this study is based on the approach developed by Amor et al. (Amor et al. 2011), adapting it to distinguish woven bone formation from lamellar bone remodeling and to introduce the impact of mechanics on biological events. In particular, a comparison of this model with the in vivo study conducted by Abrahamsson et al. was conducted to assess its relevance regarding biological events occurring during implants osseointegration and de novo bone proportions observed in implants healing chambers (Abrahamsson et al. 2004).

\subsection{Governing equations}

The present model can be divided into two main parts: a mechanical part and a biological one. The mechanical part aims at introducing the mechanical stimuli that influence cellular activity, bone formation and remodeling. Such part is modeled considering bone as a porous media filled with an interstitial fluid. This porous media is assigned with evolutive mechanical properties depending on bone regeneration and remodeling. The biological part of the model describes cells migration, proliferation and differentiation in order to produce woven bone that is further remodeled into lamellar bone. The whole biological process is, in addition to mechanical stimuli, influenced by the local concentration of growth factors.

\subsubsection{Poroelastic theory}

The mechanical part of the model is built under the hypothesis of a quasi-static domain with a negligible mass increase, and small strain. Bone tissue is composed of a solid matrix in which an insterstitial fluid flows. Therefore, we characterize bone as a biphasic material governed by poroelastic theory, considering continuous elastic and isotropic properties. Furthermore, the porous media is saturated with interstitial fluid and both solid and fluid components are considered incompressible. Total stress $\overline{\bar{\sigma}}$ in the media is therefore a function of solid matrix strain $\overline{\bar{\varepsilon}}$ and interstitial fluid pressure $p_{f}$ defined by Eq. (1), where $\mathrm{E}$ is the young modulus of the solid matrix and $\mu$ Poisson's ratio.

$\overline{\bar{\sigma}}=\frac{\nu E}{(1-2 \nu)(1+\nu)} \cdot \operatorname{Tr}(\overline{\bar{\varepsilon}}) \cdot \overline{\bar{I}}+\frac{E}{1+\nu} \overline{\bar{\varepsilon}}-p_{f} \cdot \overline{\bar{I}}$

A conservative law is used to describe the interstitial fluid speed regarding the solid strain, as defined by Eq.(2), $\phi$ and $K$ being respectively the solid phase porosity and permeability while $\mu_{f}$ and $\kappa_{f}$ are respectively the fluid dynamic viscosity and compressibility modulus. The fluid flux is related to pressure gradient through Darcy's law Eq.(3).

$$
\left(\frac{\phi}{\kappa_{f}}\right) \frac{\partial p_{f}}{\partial t}-\nabla \cdot\left(\frac{K}{\mu_{f}} \nabla p_{f}\right)=\frac{\partial}{\partial t} \operatorname{Tr}(\varepsilon)
$$


$v_{f}=-\frac{K}{\mu_{f}} \nabla p_{f}$

The implementation of the poroelastic theory is meant to calculate the distribution an adimensional criterion $\mathrm{S}$ of mechanical stimuli. This criterion derives from Prendergast et al. work on computational prediction of MSCs differentiation in fracture healing and is defined through the calculation of the octahedral shear strain $\gamma$ and interstitial fluid speed $v_{f}$ (Prendergast, P.J; Huiskes, R.; Soballe 1997):

$S=\frac{\gamma}{a}+\frac{\left|v_{f}\right|}{b}$

$a=3.75 \%$ and $b=3 \mu \mathrm{m} \cdot \mathrm{s}^{-1}$ are empirical constants (Soballe, KjeldHansenp and Bunger 1992; Søballe et al. 1992; Søballe 1993).

\subsubsection{Bone ingrowth}

The biological part of the model includes the spatio-temporal evolution of five variables: concentrations of MSCs $\left(c_{m}\right)$ and osteoblasts $\left(c_{o b}\right)$, woven bone $\left(m_{w}\right)$ and lamellar bone $\left(m_{l}\right)$ matrix densities as well as osteogenic growth factors concentration $(g)$. Compared to models developped by Andreykiv et al. (Andreykiv, van Keulen, and Prendergast 2008) or Amor et al. (Amor et al. 2011), this model does not take into account the potential generation of fibrous or cartilaginous tissues that are not normally observed in intramembraneous ossification. Therefore, the biological part of the model is represented by five differential equations of taxis-diffusion-reaction type:

$$
\begin{aligned}
\frac{\partial c_{m}}{\partial t}= & \nabla \cdot \underbrace{[\underbrace{}_{c_{m}} \nabla c_{m}}_{\text {diffusion }}-\underbrace{C_{c_{m}} c_{m} \nabla m}_{\text {haptotaxis }}-\underbrace{\chi_{c_{m}} c_{m} \nabla g}_{\text {chemotaxis }}] \\
& +\underbrace{P_{c_{m}}\left(1-\alpha c_{t o t}\right) c_{m}}_{\text {proliferation }}-\underbrace{F_{c_{o b}}\left(1-\alpha c_{o b}\right) c_{m}}_{\text {differentiation }}
\end{aligned}
$$

$$
\begin{aligned}
\frac{\partial c_{o b}}{\partial t}= & \underbrace{P_{c_{o b}}\left(1-\alpha c_{t o t}\right) c_{o b}}_{\text {proliferation }}+\underbrace{F_{c_{o b}}\left(1-\alpha c_{o b}\right) c_{m}}_{\text {differentiation }} \\
& -\underbrace{d_{c_{o b} c_{o b}}}_{\text {apoptosis }}
\end{aligned}
$$

$$
\frac{\partial m_{w}}{\partial t}=\underbrace{Q_{w}\left(1-\kappa m_{t o t}\right) c_{o b}}_{\text {secretion }}-\underbrace{Q_{l} m_{w}\left(1-\kappa m_{l}\right)}_{\text {remodeling }}
$$




$$
\frac{\partial m_{l}}{\partial t}=\underbrace{Q_{l} m_{w} m_{l}\left(1-\kappa m_{l}\right)}_{\text {remodeling }}
$$

$$
\frac{\partial g}{\partial t}=\nabla \cdot[\underbrace{D_{g} \nabla g}_{\text {diffusion }}]+\underbrace{E_{g} c_{o b}}_{\text {production }}-\underbrace{d_{g} g}_{\text {degradation }}
$$

With $c_{t o t}=c_{m}+c_{o b}$ and $m_{t o t}=m_{w}+m_{l}$. MSCs migration is considered to occur mainly through diffusion (Gruler and Bültmann 1984), chemotaxis (Lind, Eriksen, and Bünger 1996) and haptotaxis (Carter 1965). These migrations are respectively driven by a diffusion function $D_{c_{m}}$, a haptotaxis one $C_{c_{m}}$ and a chemotaxis one $\chi_{c_{m}}$. While it was demonstrated that osteoblasts have the ability to migrate towards the implant surface (Jones and Boyde 1977), this migration is limited in comparison with MSCs as osteoblasts attached to the bone surface they secrete lose this ability (Davies 2003). Therefore, osteoblasts migration is neglected regarding MSCs one. In addition to migration, MSCs and osteoblasts proliferate respectively as a function of $P_{c_{m}}$ and $P_{c_{o b}}$ that both also depend on $m_{t o t}$. MSCs differentiate depending on $F_{c_{m}}$ and osteoblasts apoptosis is considered by a simple linear relation, represented by a constant of decay $d_{c_{o} b}$. Osteoblasts concentration then influence the secretion of woven bone at a rate depending on the functional form $Q_{w}$. This woven bone subsequently remodels into lamellar bone at a rate that depends of $Q_{l}$. Except for cells migration and apoptosis, all the aforementioned functional forms depend of the stimulus $\mathrm{S}$ and/or growth factors concentration. Finally, growth factors are considered to diffuse with a constant of diffusion $D_{g}$. They are produced as a function of osteoblasts concentration at a rate $E_{g}$ and decay as a linear function of growth factors concentration at a rate $d_{g}$. An overview of all variables, parameters and functions is available in the Appendix (Tables 1, 2 and 3). In the absence of experimental data on these parameters and functions, computational models of the scientific literature are added as references to justify the values they are derived from.

\subsection{Numerical simulation}

The computational model suggested in this study was developed using COMSOL Multiphysics v5.4 software (Comsol inc, USA). It is based on a finite element formulation. An implicit method was used to solve non-linear differential equations, with a mesh closely adapted to the geometry studied. The numerical simulation is conducted in 12 iterative steps to cover twelves weeks of peri-implant bone healing (Fig. 1). At each iteration, the spatio-temporal distribution of each variable is stored as well as their proportion in the region of bone healing. It is to notice that biological activity was only computed in areas concerned by bone healing. Before simulating the osseointegration of different thread design, this model was calibrated by comparison with data from scientific literature (Berglundh et al. 2003; Abrahamsson et al. 2004).

\subsubsection{Model geometries}

For time computation and simplification matters, 2D geometries containing one half thread and one half screw healing chamber were simulated in this study. Symme- 
try conditions were applied in accordance with such simplification. Each geometry implemented into the numerical simulation was subdivided into different domains, representing a titanium screw implanted in a trabecular bone environment (Fig. 2a). For each screw profile, a pre-drilled hole is modeled. The pre-drilled hole and implant dimensions altogether constrain the healing chamber size. Furthermore, to model bone damage that is initially obtained through drilling and implant insertion (Brunski 1999; Kuzyk and Schemitsch 2011), a damaged bone area was implemented distinguishing the bone healing chamber domain from healthy bone by a zone of $450 \mu \mathrm{m}$ thick (Kuzyk and Schemitsch 2011). x-axis geometries limits were constrained on one side by the screw axis and was set to twice the external implant radius on the other side.

\subsubsection{Material properties}

Three materials, representing three tissue state of maturity and mineralization were defined in this study: granulation tissue, woven immature bone and lamellar mature bone. Mechanical properties of these materials are summarized in Table 4. As significantly more resistant than bone, the titanium implant is considered as an infinitely rigid material in the context of our study. Evolutive materials properties were applied to the healing chamber and damaged bone domains, using a law of mixture for each property considered:

$E=\left(1-\kappa m_{\text {tot }}\right) E_{\text {gran }}+\kappa\left(m_{w} E_{w}+m_{l} E_{l}\right)$

Evolutive materials properties are updated at each simulation iteration. Finally, healthy bone was considered as mature bone and assigned with properties of lamellar bone.

\subsubsection{Initial values and boundary conditions}

Initially, the healing chamber is considered filled with a very soft granulation tissue, while the healthy bone is only composed of trabecular bone. To model the initial bone resorption following bone drilling and implant insertion, trabecular bone is considered reduced by half in the damaged bone area (Timon and Keady 2019). The implant is studied in an unloaded configuration. Mechanical boundary conditions are applied at the external limit (Fig. 2b) to model an external stimulation of bone tissue. At this limit, the interstitial fluid pressure was considered null. Therefore, a Dirichlet condition was applied such as $p_{f}=0 \mathrm{~Pa}$ at this limit. Furthermore, to introduce the influence of a mechanical stimulus on cellular activity, a compressive displacement $U_{0}=0.5 \mu \mathrm{m}$ was applied at the external limit of the model. Such displacement was chosen in order to induce a moderate stress and pressure inside the bone healing chamber. To observe cellular activity variations regarding the mechanical stimulus, several studies were conducted on one thread profile $\left(\mathrm{r}_{\text {int }}=3 \mathrm{~mm}, \mathrm{e}=0.06 \mathrm{~mm}\right.$ and a $=30^{\circ}$ ) with increasing displacements from $0.25 \mu \mathrm{m}$ to $1.25 \mu \mathrm{m}$. As the titanium implant was considered infinitely rigid, boundaries at the interface with the implant healing chamber and with damaged bone were fixed. Then, symmetry conditions were applied at upper and lower limits to model the screws thread repetition. Biological boundary conditions were applied at the interface between damaged bone and healthy bone (Fig. 2b). Dirichlet conditions were applied for MSCs and growth factors concentrations, such as $c_{m_{0}}=10^{5}$ cells.ml ${ }^{-1}$ and $g_{0}=10 \mathrm{ng} \cdot \mathrm{ml}^{-1}$. Cellular and growth factors constant 
provision were respectively considered to occur respectively during 4 and 10 (Amor et al. 2011) and cells proliferation during 7 days (Schmitt 2015).

\subsubsection{Model calibration regarding scientific literature}

In order to provide results that can be compared with literature, a model calibration was conducted. Such calibration was based on a study conducted by Abrahamsson et al. (2004) in vivo (Berglundh et al. 2003; Abrahamsson et al. 2004). This study aimed at evaluating the rate and degree of osseointegration of dental implants during early phases of healing. Such evaluation was conducted in dogs jaw and for two implants topographies: a standard surface with no surface treatment turned and a microrough topography obtained through sandblasting and acid etching. The geometry modeled for this calibration was derived from the geometry of the titanium implants used for this study. Therefore, an implant with an external diameter of $4.1 \mathrm{~mm}$, an internal diameter of $2.6 \mathrm{~mm}$, a pitch of $1.25 \mathrm{~mm}$ and inserted into a pre-drilled hole of $3.4 \mathrm{~mm}$ diameter was considered for this simulation, resulting in a $0.4 \mathrm{~mm}$ deep healing chamber (Fig. 3a,b). The healing chamber and its environment have been modeled using the domains subdivision described previously. Considering the mathematical modeled developped and the available data from the literature to set a value for each parameter (Table 1), The calibration was conducted by varying the parameters related to woven bone secretion $\alpha_{w}$ and lamellar bone remodeling $Q_{l_{0}}$. The validation is based on the comparison of woven bone and lamellar bone proportions within the implant healing chamber, comparing the turned implant experimental data and numerical simulation results. The most faithful results obtained with $\alpha_{w}=2.5 \times 10^{-4}$ g.day ${ }^{-1}$ and $Q_{l_{0}}=0.9 \times 10^{-6} \mathrm{~mm}^{3}$.(g.day) ${ }^{-1}$ are displayed in this article.

\subsubsection{Parametric analysis}

A parametric analysis was conducted in order to study the impact of the implant geometry on the osseointegration process that has been modeled. Titanium screws commonly used in the orthopedic field with an external diameter $4.5 \mathrm{~mm}$, a pitch of $1.75 \mathrm{~mm}$ and inserted into a pre-drilled hole of $2 \mathrm{~mm}$ diameter were considered in this analysis. Three parameters were considered to define the healing chamber geometry: the thread thickness $e$, the thread angle $a$ and the core radius $r_{i n t}$ of the implant (Fig. $3 \mathrm{c}, \mathrm{d})$. The range of values studied for each parameter is indicated in Table 5.

\subsubsection{Surface treatment modeling}

For the same thread profile that was used for the analysis of mechanical stimuli variations, simulations were conducted with local modifications of governing equations and initial conditions in order to model an implant surface treatment. Therefore, no modifications have been conducted on the implant macrogeometry nor its microgeometry and the surface treatment was simulated through the local modification of the cellular activity, based on data from the literature (Lincks et al. 1998). At the surface treatment interface (domains 5 and 6 in Fig. 2a), cells proliferation, differentiation and apoptosis, as well as woven bone formation rate and growth factors production rate were modulated through weighting coefficients according to data obtained in vitro from Lincks et al. (Lincks et al. 1998) (Table 6). Furthermore, to model the impact of the surface treatment on the initial blood proteins adsorption, a Dirichlet condition for growth factors concentration was set at this same interface such as $\mathrm{g}_{0 i}=2 \mu \mathrm{g}$. $\mathrm{ml}^{-1}$ (Fig. 2c), which remains in the order of values of protein concentrations adsorbed on 
titanium surfaces (Sela et al. 2007).

\subsubsection{Mesh settings}

Depending on the geometry and the boundary counditions that were simulated, mesh settings were varied to provide the convergence of the simulation. Regarding the model calibration, triangular elements with a size from $3.62 \times 10^{3} \mathrm{~mm}$ to $0.17 \mathrm{~mm}$ were used, for a total of 11250 elements and an average element quality (skewness) of 0.8989. On the other hand, triangular element sizes from $1.12 \times 10^{4} \mathrm{~mm}$ to $0.2 \mathrm{~mm}$ were used for a total number ranging from 15827 to 59801 elements and an average element quality of 0.9237 . Finally, 72009 quadrilateral elements with a size from $4.93 \times 10^{4} \mathrm{~mm}$ to $0.26 \mathrm{~mm}$ were used for the surface treatment simulation mesh, with an average element quality of 0.937 . In order to verify the mesh size does not influence simulation results, a convergence analysis was conducted by varying the elements size by $\pm 50 \%$. No modification of the final results through the convergence analysis were reported by the authors in terms of bone densities.

\section{Results}

\section{1. $\quad$ Model calibration with experimental findings}

Regarding cellular activity, we observed that the damaged bone area is rapidly filled with growth factors and MSCs differentiating into osteoblasts (Fig. 4). From week 1 to week 4, cells keep migrating from host bone towards the surface of the implants within the healing chamber. Cells concentration further decreased slowly until week 12 . Regarding woven bone and lamellar bone formation, a front of woven bone formation was observed going from host bone towards the surface of the implant. This woven bone is rapidly replaced by lamellar bone as and when the front progresses. These results are well-correlated with peri-implant bone healing events that were emphasized by Abrahamsson et al., as a woven bone matrix forms between weeks 0 and 4, that is progressively remodeled into lamellar bone (Fig. 5) (Abrahamsson et al. 2004). In particular, an increase in mineralization rate is visible between week 4 and 6 before slowing down from week 8 . In terms of quantitative results, lamellar bone proportions results accurately fit the experimental data. On the other hand, woven bone simulation results slightly diverge from experimental data. While a peak in woven bone proportion is reached experimentally from week 2 , simulation results predicted a slower formation, with a peak reached at week 6 .

\subsection{Parametric analysis}

While revealing no differences in the sequence of biological events occurring during the simulation, change applied in thread dimensions had an impact in the proportions of woven bone and lamellar bone observed (Fig. 6a, b). Considering extreme values of parameters, significant differences were found between an implant defined such as $\mathrm{r}_{\text {int }}=1.5 \mathrm{~mm}, \mathrm{e}=0.06 \mathrm{~mm}, \mathrm{a}=40^{\circ}$ and the one with $\mathrm{r}_{\text {int }}=1 \mathrm{~mm}, \mathrm{e}=0.4 \mathrm{~mm}, \mathrm{a}=0^{\circ}$. At week 12 , the parametric analysis highlighted the major effect of the implant internal radius regarding the two other parameters, showing lower woven bone proportions (Fig. 6c, e) within the healing chamber and higher lamellar bone one (Fig. 6d, f) with decreasing internal radius. Similarly, decreasing the thread thickness tends to generate a decrease 
in woven bone proportion and an increase in lamellar bone one. These evolutions occurred however to a lesser extent than those modifying the internal radius. Finally, it was observed in our simulations that modifying the thread angle does not have a significant impact on the biological response.

\subsection{Influence of mechanical stimuli}

Mechanical stimuli applied were found to have an impact on relative bone formation. Indeed, increasing the applied displacement from $0.25 \mu \mathrm{m}$ to $1.25 \mu \mathrm{m}$ generated an up to 5 -fold decrease in woven bone formation at week 4 (Fig. 7a). On the other hand, lamellar bone evolution with increasing displacement is more discrete, showing up to a 1.35-fold increase in lamellar bone formation at the same time period (Fig. 7b).

\section{4. $\quad$ Surface treatment}

The local changes applied on cells activity, growth factors production as well as bone formation changed the pattern of the global biological behavior (Fig. 8). Indeed, the deposition of osteoblasts and growth factors onto the implant surface in week 2 generated marked signs of osteoconduction through woven bone formation from the implant surface towards host bone that could be observed at week 4 . Just like the untreated surface, a decrease of growth factor and osteoblasts were observed within the healing chamber from week 4 . Regarding woven bone and lamellar bone proportions within the healing chamber, different early behaviors were noticed compared to the untreated surface. A peak in woven bone was observed at week 4 (Fig. 9a), at which the value reached 3 times the one of the untreated implant. Likewise, lamellar bone showed at week 4 a 2.5 times lower proportion than the untreated surface at the same time period (Fig. 9b).

\section{Discussion}

The model presented in this study, through the evaluation of different parameters influence, highlighted several major phenomenon occurring during peri-implant bone healing. On the one hand, it is to notice the ability of the model to reproduce woven bone and lamellar bone formation trends from the work of Abrahamsson et al. (Abrahamsson et al. 2004). Quantitative simulations results successfully reached the same order of values than the ones measured in vivo within the healing chamber. In comparison with other attempts to model this behavior, Amor et al. (2011) showed faster bone formation in their simulations compared to experiments (Amor et al. 2011). Considering the parametric analysis, our study evaluated the impact of the thread thickness, the thread angle and the implant internal radius on osseointegration. Among these three parameters, our results suggest that the implant internal radius has a preponderant role in woven bone formation and the following bone remodeling into lamellar bone. These results are in accordance with the initial observation of Lemons et al. (2000) (Lemons 2000) that was further validated by various studies (Coelho et al. 2010; Leonard et al. 2009). Indeed, these studies pointed out the greater woven bone formation with deeper healing chamber compared to implants allowing no space between its core and host bone. By comparing several healing chamber configuration, Marin et al. (2010) also denoted that woven bone only filled half of the deepest implants' healing 
chambers, resulting in lower BIC compared to implants with smaller healing chambers (Marin et al. 2010). Regardless of the impact on primary stability achievement, our model suggests a faster peri-implant healing with increasing implant internal radius. On the other hand, it is known that the relative motion between an implant and host bone affects peri-implant bone ingrowth (Davies 2003). While a low to moderate level of mechanical stimulation positively affects bone cells response, studies also reported the existence of a threshold above which the motion becomes excessive and leads to the formation of fibrous tissue. Different threshold levels have been used in computational models, allowing maximum bone ingrowth for micromotions of $20 \mu \mathrm{m}$ (Andreykiv et al. 2005) or $40 \mu \mathrm{m}$ (Spears et al. 2000). In the absence of sufficient scientific proof, it is yet to admit that the appropriate mechanical stimulation of bone formation remains unknown, especially since this level might also be specy-specific. Therefore, computational models could only provide an insight of mechanically stimulated peri-implant bone healing. In our case, local and low values from $0.25 \mu \mathrm{m}$ to $1.25 \mu \mathrm{m}$ were applied as a boundary condition in order to assess the sensitivity of this parameter on bone formation. In this model, the mechanical stimulation was considered by computing the octahedric shear strain and the interstitial fluid speed, gathered into the Prendergast criterion. Although this criterion was initially used to predict fracture healing, it remains one the most faithful criteria among the scientific literature (Lacroix and Prendergast 2002; Isaksson et al. 2006), even in the case of peri-implant osteogenesis. The surface treatment model is able to portray several major phenomena compared to untreated implants, although discrepancies remain between simulations results and experimental findings. First, the peak of woven bone formation observed in vivo by Abrahamsson et al. (2004) is correctly predicted by the model (Abrahamsson et al. 2004). Nonetheless, the temporal occurrence of this peak as well as its magnitude differ, as the maximum woven bone proportion obtained through the model at week 4 reaches half the experimentally measured one at week 2 . Beyond the differences between implants' dimensions for this simulation, this discrepancy might be explained by the fact that, in absence of sufficient data from the literature, weighting coefficient used to model a surface treatment were taken from the study conducted by Lincks et al. (1998) (Lincks et al. 1998). Although the roughness reported by this study is similar to the SLA surface treatment, it is more likely that the cellular response induced in vitro on these surfaces differs from the one obtained in vivo on a surface that may have other chemical or topographical properties, resulting in such prediction bias. Then, our model correctly highlights osteoconduction and the formation of a layer of bony tissue at the surface of the implant. Similar observations were made in vivo, depicting the formation of a bone shell around rough and porous surfaces (Gross et al. 1990; Perrin et al. 2002). Finally, the model does not reproduce the subsequent trabecularization that has been observed in the work of Perrin et al. (Perrin et al. 2002). This architecture rearrangement is obtained through a mechanically driven combination of bone secretion and bone resorption, functionally adapting bone structure to the implant in order to optimize its response to mechanical stress. In our case, this optimization was not modeled due to the computational power requirements such implementation requires. The absence of bone architecture reorganization might explain the lower proportions of lamellar bone observed in the literature within the healing chamber (Abrahamsson et al. 2004). More generally, while this model allows to reproduce peri-implant healing patterns and to corroborate the influence of mechanical strain combined with biological factors, as well as the influence of surface treatment on cellular activity and tissue production, it remains a simplification of the actual phenomena at stake. Several improvements can be suggested, assuming the availability of 
sufficient computation power. On the one hand, the osseointegration process was simulated without taking into account the vascular events occuring through angiogenesis. Indeed, it is known through the scientific literature that the implant design and its surface treatment have an impact on the formation of surrounding new vessels(Khayat et al. 2013; Saghiri et al. 2016). In particular, pericytes were shown to have an important role in migration of osteoblasts towards the healing zone (Murray et al. 2014). In our model, such multipotents cells were concatenated into the mesenchymal stem cells variable that results in a simplification from which may arise interpretation bias. Further improvements could be conducted by considering cells phenotypes individually in order to simulate their interaction in the osseointegration process. On the other hand, bone was considered as a continuous elastic and isotropic material. Although this simplification is used in many papers and allows to save computation time (Vulović and Filipovic 2020), bone was shown experimentally to be a viscoelastic and anisotropic material (Turner et al. 1999). Further development will have to take these properties into account in order to increase the accuracy of the model. Then, load conditions were considered to be represented by a compressive displacement applied at the external limit of the model. This displacement was assumed considering surgical protocols applied in scientific literature, implying that no crown was added to the implants. Therefore, forces obtained through chewing would only be applied from surrounding host bone to the implant. Nonetheless, this approach of the mechanical load remains an arbitrary simplification of the exact implant loading conditions. Considering a 3D geometry of an implant inserted into a mandible may allow to set more faithful loading conditions for the simulation. Moreover, a rigid bond between the implant and bony tissue was considered in this study. Yet, It appears that a close relation exists between implants osseointegration and the primary stability that is achieved (Javed et al. 2013). Indeed, thread patterns as well as the surgical procedure for their insertion induce an initial stress distribution in bone. Such distribution act as a mechanical stimulation for osteogenic cells, that alters their behavior positively or negatively if the implant overloads surrounding tissues. Furthermore, the achievement of a correct primary stability lies in the occurence of minimal micromotion between the implant and tissues at its interface. Such micromotion could be introduced in further work by considering the implant in its larger environment and adding the mechanical impact of implant insertion into bone. Finally, this model only considered a healthy case of periimplant healing. Indeed, no attempt was made to model the mesenchymal stem cells differentiation into fibroblasts nor chondroblasts in case of higher mechanical stimulation, according to Prendergast criterion. Therefore, this work could be completed in the future with the eventual production of other tissue phenotypes such as fibrous tissue or cartilage by adapting Prendergast et al. mechanical criterion, at the price of the addition of more complexity and mathematical non-linearities to the model. In conclusion, the model developped in this study brings a first approach of an efficient method to study the osseointegration process in an ehtical and cost-effective way. Furthermore, many variables of mechanical or biological nature can be included in the model, providing a method to incrementally add complexity to the simulations that are conducted. Potential clinical benefits are numerous, from dental applications to orthopedic and traumatologic ones. First, the improvement of this model could provide a better understanding of the phenomena occuring during the osseointegration process, such as angiogenesis or the impact of biomechanical aspects on the process of bone remodeling. Then, this model could help optimizing implants design by bringing ways to study the influence of their geometry and mechanical properties on cellular activity, as well as the ability to simulate passive or bioactive surface treatments. 


\section{Conflict of Interest}

No potential conflict of interest was reported by the authors.

\section{References}

Abrahamsson, Ingemar, Tord Berglundh, Elena Linder, Niklaus P. Lang, and Jan Lindhe. 2004. "Early bone formation adjacent to rough and turned endosseous implant surfaces. An experimental study in the dog." Clinical Oral Implants Research 15 (4): 381-392.

Ambard, Dominique, Gaëtan Guérin, and Pascal Swider. 2009. "A reactive poroelastic model to predict the periprosthetic tissue healing." European Journal of Computational Mechanics 18 (1): 131-143.

Ambard, Dominique, and Pascal Swider. 2006. "A predictive mechano-biological model of the bone-implant healing." European Journal of Mechanics, A/Solids 25 (6): 927-937.

Amor, N., L. Geris, J. Vander Sloten, and H. Van Oosterwyck. 2011. "Computational modelling of biomaterial surface interactions with blood platelets and osteoblastic cells for the prediction of contact osteogenesis." Acta Biomaterialia 7 (2): 779-790. http://dx.doi.org/10.1016/j.actbio.2010.09.025.

Andreykiv, A., P. J. Prendergast, F. Van Keulen, W. Swieszkowski, and P. M. Rozing. 2005. "Bone ingrowth simulation for a concept glenoid component design." Journal of Biomechanics 38 (5): 1023-1033.

Andreykiv, A., F. van Keulen, and P. J. Prendergast. 2008. "Computational Mechanobiology to Study the Effect of Surface Geometry on Peri-Implant Tissue Differentiation." Journal of Biomechanical Engineering 130 (5): 051015.

Bailón-Plaza, Alicia, and Marjolein C.H. Van Der Meulen. 2001. "A mathematical framework to study the effects of growth factor influences on fracture healing." Journal of Theoretical Biology 212 (2): 191-209.

Berglundh, Tord, Ingemar Abrahamsson, Niklaus P Lang, and Jan Lindhe. 2003. "De novo alveolar bone formation adjacent to endosseous implants." Clinical oral implants research 14 (3): 251-262.

Brånemark, P. I., U. Breine, R. Adell, B. O. Hansson, J. Lindström, and A. Ohlsson. 1969. "Intra-osseous anchorage of dental prostheses: I. Experimental studies." Scandinavian Journal of Plastic and Reconstructive Surgery and Hand Surgery 3 (2): 81-100.

Brunski, J. B. 1999. "In vivo bone response to biomechanical loading at the bone/dentalimplant interface." Advances in dental research 13: 99-119.

Carter, S. B. 1965. "C 1965 Nature Publishing Group." Nature 206: 757-761.

Coelho, Paulo G., Marcelo Suzuki, Marcia V M Guimaraes, Charles Marin, Rodrigo Granato, Jose N. Gil, and Robert J. Miller. 2010. "Early bone healing around different implant bulk designs and surgical techniques: A study in dogs." Clinical Implant Dentistry and Related Research 12 (3): 202-208.

Coffey, Robert J, William E Russell, and John A Barnard. 1990. "Pharmacokinetics of TGFP with Emphasis on Effects in Liver and Gut." Annals of the New York Academy of Sciences 285-291.

Davies, J. 2003. "Understanding peri-implant endosseous healing." Journal of dental education 67 (8): 932-949.

Geris, Liesbet, Alf Gerisch, Jos Vander Sloten, Rüdiger Weiner, and Hans Van Oosterwyck. 2008. "Angiogenesis in bone fracture healing: A bioregulatory model." Journal of Theoretical Biology 251 (1): 137-158.

Gittens, Rolando A., Rene Olivares-Navarrete, Alice Cheng, David M. Anderson, Taylor McLahan, Ingrid Stephan, Jürgen Geis-Gerstorfer, et al. 2013. "The Roles of Titanium Surface Micro/Nanotopography and Wettability on the Differential Response of Human Osteoblast Lineage Cells." Acta Biomaterialia 9 (4): 6268-6277. 
Gross, U., C. Müller-Mai, T. Fritz, C. Voigt, W. Knarse, and H. J. Schmitz. 1990. "Implant surface roughness and mode of load transmission influence peri implant bone structure." Clinical Implant Materials (Advances in Biomaterials) 9: 303-308.

Gruler, H, and B D Bültmann. 1984. "Analysis of cell movement." Blood cells 10 (1): 61-77. http://www.ncbi.nlm.nih.gov/pubmed/6487816.

Isaksson, Hanna, Wouter Wilson, Corrinus C. van Donkelaar, Rik Huiskes, and Keita Ito. 2006. "Comparison of biophysical stimuli for mechano-regulation of tissue differentiation during fracture healing." Journal of Biomechanics 39 (8): 1507-1516.

Javed, Fawad, Hameeda Bashir Ahmed, Roberto Crespi, and Georgios E. Romanos. 2013. "Role of primary stability for successful osseointegration of dental implants: Factors of influence and evaluation." Interventional Medicine and Applied Science 5 (4): 162-167. http://www.akademiai.com/doi/abs/10.1556/IMAS.5.2013.4.3.

Jones, S. J., and A. Boyde. 1977. "The migration of osteoblasts." Cell and Tissue Research $184(2): 179-193$.

Joyce, Michael E, Anita B Roberts, Michael B Sporn, and Mark E Bolander. 1990. "Transforming Growth Factor-Beta nd the Initiation of Chondrogenesis and Osteogenesis in the Rat Femur." 110 (June): 2195-2207.

Kapur, Sonia, David J Baylink, and K William Lau. 2003. "Fluid flow shear stress stimulates human osteoblast proliferation and differentiation through multiple interacting and competing signal transduction pathways." Bone 32: 241-251.

Kaspar, D., W. Seidl, C. Neidlinger-Wilke, A. Ignatius, and L. Claes. 2000. "Dynamic cell stretching increases human osteoblast proliferation and CICP synthesis but decreases osteocalcin synthesis and alkaline phosphatase activity." Journal of Biomechanics 33 (1): 45-51.

Khayat, Philippe G., Hélène M. Arnal, Bahige I. Tourbah, and Lars Sennerby. 2013. "Clinical outcome of dental implants placed with high insertion torques (Up to $176 \mathrm{Ncm})$." Clinical Implant Dentistry and Related Research 15 (2): 227-233.

Kuzyk, Paul R.T., and Emil H. Schemitsch. 2011. "The basic science of peri-implant bone healing." Indian Journal of Orthopaedics 45 (2): 108-115.

Lacroix, D., and P. J. Prendergast. 2002. "A mechano-regulation model for tissue differentiation during fracture healing: Analysis of gap size and loading." Journal of Biomechanics 35 (9): 1163-1171.

Lemons, J.E. 2000. "Structure of bone adjacent to different dental implants." In Bone Engineering, edited by Em Squared Inc., 313-321. Toronto.

Leonard, Gary, Paulo Coelho, Ioannis Polyzois, Leo Stassen, and Noel Claffey. 2009. "A study of the bone healing kinetics of plateau versus screw root design titanium dental implants." Clinical Oral Implants Research 20 (3): 232-239.

Lincks, J, B D Boyan, C R Blanchard, C H Lohmann, Y Liu, D L Cochran, D D Dean, and Z Schwartz. 1998. "Response of MG63 osteoblast-like cells to titanium and titanium alloy is dependent on surface roughness and composition." Biomaterials 19: 2219-2232.

Lind, M., E.F. Eriksen, and C. Bünger. 1996. "Bone Morphogenetic Protein-2 but not Bone Morphogenetic Protein-4 and -6 Stimulates Chemotactic Migration of." Bone 18 (1): 53-57.

Marin, Charles, Rodrigo Granato, Marcelo Suzuki, Jose N. Gil, Malvin N. Janal, and Paulo G. Coelho. 2010. "Histomorphologic and histomorphometric evaluation of various endosseous implant healing chamber configurations at early implantation times: A study in dogs." Clinical Oral Implants Research 21 (6): 577-583.

Martino, Fabiana, Ana R. Perestrelo, Vladimír Vinarský, Stefania Pagliari, and Giancarlo Forte. 2018. "Cellular mechanotransduction: From tension to function." Frontiers in Physiology 9 (JUL): 1-21.

Mavrogenis, A. F., R. Dimitriou, J. Parvizi, and George C. Babis. 2009. "Biology of implant osseointegration." Journal of Musculoskeletal Neuronal Interactions 9 (2): 61-71.

Meyer, U., U. Joos, J. Mythili, T. Stamm, A. Hohoff, T. Fillies, U. Stratmann, and H. P. Wiesmann. 2004. "Ultrastructural characterization of the implant/bone interface of immediately loaded dental implants." Biomaterials 25 (10): 1959-1967.

Moreo, Pedro, José Manuel García-Aznar, and Manuel Doblaré. 2009. "Bone ingrowth on the 
surface of endosseous implants. Part 1: Mathematical model." Journal of Theoretical Biology 260 (1): 1-12.

Murray, Iain R., Christopher C. West, Winters R. Hardy, Aaron W. James, Tea Soon Park, Alan Nguyen, Tulyapruek Tawonsawatruk, Lorenza Lazzari, Chia Soo, and Bruno Péault. 2014. "Natural history of mesenchymal stem cells, from vessel walls to culture vessels." Cellular and Molecular Life Sciences 71 (8): 1353-1374.

Olsen, Luke, Jonathan A. Sherratt, Philip K. Maini, and Frank Arnold. 1997. "A mathematical model for the capillary endothelial cell-extracellular matrix interactions in wound-healing angiogenesis." IMA Journal of Mathemathics Applied in Medicine and Biology 14 (4): 261281.

Park, Jung, Sebastian Bauer, Karl Andreas Schlegel, Friedrich W. Neukam, Klaus Der Von Mark, and Patrik Schmuki. 2009. "TiO2 nanotube surfaces: $15 \mathrm{~nm}$ - an optimal length scale of surface topography for cell adhesion and differentiation." Small 5 (6): 666-671.

Perrin, Daniel, Serge Szmukler-Moncler, Casimir Echikou, Philippe Pointaire, and Jean Pierre Bernard. 2002. "Bone response to alteration of surface topography and surface composition of sandblasted and acid etched (SLA) implants." Clinical Oral Implants Research 13 (5): 465-469.

Prendergast, P.J; Huiskes, R.; Soballe, K.;. 1997. "Biophysical stimuli on cells during tissue differentiation at implant interfaces." Journal of biomechanics 30 (6): 539-548. http://www.sciencedirect.com/science/article/pii/S0021929096001406.

Saghiri, Mohammad Ali, Armen Asatourian, Franklin Garcia-Godoy, and Nader Sheibani. 2016. "The role of angiogenesis in implant dentistry part I: Review of titanium alloys, surface characteristics and treatments." Medicina Oral, Patologia Oral y Cirugia Bucal 21 (4): e514-e525.

Schmitt, Mary. 2015. "Analyses expérimentales et modélisation numérique de l'ostégenese au sein d 'un implant poreux en titane." Thèse : Ecole Nationale Supérieure d'Arts et Métiers https://pastel.archives-ouvertes.fr/tel-01179783.

Schroeder, A. C., Ortrun E. M. Pohler, and Franz Sutter. 1976. "Tissue reaction to an implant of a titanium hollow cylinder with a titanium surface spray layer." SSO Schweiz Monatsschr Zahnheilkd 86 (7): 713-27.

Schwartz, Zvi, Perry Raz, Ge Zhao, Yael Barak, Michael Tauber, Hai Yao, and Barbara D. Boyan. 2008. "Effect of micrometer-scale roughness of the surface of Ti6Al4V pedicle screws in vitro and in vivo." Journal of Bone and Joint Surgery - Series A 90 (11): 2485-2498.

Sela, Michael N., Liad Badihi, Graciela Rosen, Doron Steinberg, and David Kohavi. 2007. "Adsorption of human plasma proteins to modified titanium surfaces." Clinical Oral Implants Research 18 (5): 630-638.

Shiffler, Kyle, David Lee, Mark Rowan, Tara Aghaloo, Joan Pi-Anfruns, and Peter K. Moy. 2016. "Effect of length, diameter, intraoral location on implant stability." Oral Surgery, Oral Medicine, Oral Pathology and Oral Radiology 122 (6): e193-e198.

Simmons, Craig A, Sean Matlis, Amanda J Thornton, Shaoqiong Chen, Yu Wang, and David J Mooney. 2003. "Cyclic strain enhances matrix mineralization by adult human mesenchymal stem cells via the extracellular signal-regulated kinase ( ERK1 / 2 ) signaling pathway." 36: 1087-1096.

Søballe, Kjeld. 1993. "Hydroxyapatite ceramic coating for bone implant fixation: Mechanical and histological studies in dogs." Acta Orthopaedica 64 (S255): 1-58.

Søballe, Kjeld, Ebbe S. Hansen, Helle B.-Rasmussen, Peter H. Jørgensen, and Code Bünger. 1992. "Tissue ingrowth into titanium and hydroxyapatite-coated implants during stable and unstable mechanical conditions." Journal of Orthopaedic Research 10 (2): 285-299.

Soballe, KjeldHansenp, Ebbe Stender, and Cody Bunger. 1992. "Hydroxyapat i te coating mod if ies implant membrane formation ,." Acta orthopaedica Scandinavica 63 (2): 128-140.

Song, Guanbin, Yang Ju, Xiaodong Shen, Qing Luo, Yisong Shi, and Jian Qin. 2007. "Mechanical stretch promotes proliferation of rat bone marrow mesenchymal stem cells." Colloids and Surfaces B: Biointerfaces 58 (2): 271-277.

Spears, Iain R., Martin Pfleiderer, Erich Schneider, Ekkehard Hille, Georg Bergmann, and 
Michael M. Morlock. 2000. "Interfacial conditions between a press-fit acetabular cup and bone during daily activities: Implications for achieving bone in-growth." Journal of Biomechanics 33 (11): 1471-1477.

Szmukler-Moncler, S., D. Perrin, V. Ahossi, G. Magnin, and J. P. Bernard. 2004. "Biological Properties of Acid Etched Titanium Implants: Effect of Sandblasting on Bone Anchorage." Journal of Biomedical Materials Research - Part B Applied Biomaterials 68 (2): 149-159.

Timon, Charles, and Conor Keady. 2019. "Thermal Osteonecrosis Caused by Bone Drilling in Orthopedic Surgery: A Literature Review." Cureus 11 (7): 1-7.

Turner, Charles H., Jae Rho, Yuichi Takano, Ting Y. Tsui, and George M. Pharr. 1999. "The elastic properties of trabecular and cortical bone tissues are similar: Results from two microscopic measurement techniques." Journal of Biomechanics 32 (4): 437-441.

Vander, A., J. Sherman, and D. Luciano. 1998. Human physiology: the mechanics of body function. Ma: Wcb mc ed. Boston.

Vulović, Aleksandra Z., and Nenad Filipovic. 2020. The biomechanics of lower human extremities.

Weyts, F. A.A., B. Bosmans, R. Niesing, J. P.T.M. Van Leeuwen, and H. Weinans. 2003. "Mechanical control of human osteoblast apoptosis and proliferation in relation to differentiation." Calcified Tissue International 72 (4): 505-512.

Zhuang, Hongming, Wei Wang, A. David Tahernia, Craig L. Levitz, Wayne T. Luchetti, and Carl T. Brighton. 1996. "Mechanical strain-Induced proliferation of osteoblastic cells parallels increased TGF- $\beta 1$ mRNA." Biochemical and Biophysical Research Communications 229 (2): 449-453.

\section{Appendix A. Model parameters rational}

The estimation of parameters used for the present model were based on several in vitro studies (Gruler and Bültmann 1984; Joyce et al. 1990), other computational models developed peri-implant bone healing (Bailón-Plaza and Van Der Meulen 2001; Andreykiv, van Keulen, and Prendergast 2008; Amor et al. 2011) and on calibration conducted using experimental data from an in vivo study (Berglundh et al. 2003; Abrahamsson et al. 2004). While few parameters were taken from computational models on fracture healing, it was reported similarities between this last ossification process and peri-implant bone-healing (Davies 2003). A summary of models parameters and functional forms is a available in Table 1 and Table 2.

- Mesenchymal stem cells: MSCs migration coefficient were taken from Andreykiv et al. work (Andreykiv, van Keulen, and Prendergast 2008), based on a leukocyte movement in vitro study (Gruler and Bültmann 1984). Diffusion coefficient was set to $D_{h}=0.0126 \mathrm{~mm}^{2}$.day ${ }^{-} 1$, which is in accordance with order of values also used in other mechanoregulatory models. While receptor-kinetic forms were used by many computational studies (Bailón-Plaza and Van Der Meulen 2001; Amor et al. 2011), simpler linear formulation were used in this study to model haptotaxis and chemotaxis, migration rates decreasing with an increase respectively in ECM matric and growth factors concentration. $C_{c_{m_{0}}}=$ $1.224 \mathrm{~mm}^{2}$.day ${ }^{-1}$. $\left(\mathrm{g} \cdot \mathrm{ml}^{-1}\right)$ and $\chi_{c_{m_{0}}}=0.027 \mathrm{~mm}^{2}$.day ${ }^{-1}$. (ng. $\mathrm{ml}^{-1}$ ) were therefore adapted from Amor et al. parameter values taking into account this simplification (Amor et al. 2011). Mechanical stimulus influence on MSCs proliferation is based on Simmons et al. in vitro study (Simmons et al. 2003). According to there work, mechanical strain negatively affects MSCs proliferation. A controversy remains however about this behavior as other studies report an increase of 
MSCs proliferation (Song et al. 2007). Therefore, Andreykiv et al. formulation as a decreasing linear function of mechanical stimulus was used in this model, with a maximum proliferation of 1.2 day $^{-1}$ reported (Andreykiv, van Keulen, and Prendergast 2008; Simmons et al. 2003). Such proliferation as well as osteoblasts one was not considered dependent of growth factors concentration, as depicted by Zhuang et al. work on mechanical-induced osteogenic cells (Zhuang et al. 1996). $F_{c_{o b}}$ functional form was defined such as both an increase of growth factors concentration and mechanical stimulus are in favor of MSCs differentiation into osteoblasts. In the absence of data from scientific literature experimental work, $\alpha_{o b}=10$ day $^{-1}$ and $\beta_{o b}=100$ day $^{-1}$ were chosen chosen with the same order of values of Baílon-Plaza et al. model (Bailón-Plaza and Van Der Meulen 2001), while $F_{c_{o b_{0}}}=0.15$ day $^{-1}$ was derived from Andreykiv et al. (Andreykiv, van Keulen, and Prendergast 2008), assuming that MSCs differentiation into osteoblasts is maximum at the mechanical stimulus above which MSCs start to differentiate into chondrocytes (Prendergast, P.J; Huiskes, R.; Soballe 1997).

- Osteoblasts: functional form defining osteoblasts proliferation was taken from Andreykiv et al. (Andreykiv, van Keulen, and Prendergast 2008), based on in vitro observations of rates ranging from 0.5 day $^{-1}$ to 1 day $^{-1}$ with a 1.5 times increase in function of mechanical stimulation (Kapur, Baylink, and Lau 2003; Kaspar et al. 2000; Weyts et al. 2003). This proliferation is assumed maximum at the mechanical stimulus above which MSCs start to differentiate into chondrocytes as well. Finally, osteoblast apoptosis rate $d_{c_{o b}}=0.1$ day ${ }^{-1}$ was chosen in accordance with the results a linear stability analysis conducted by Baílon-Plaza et al (Bailón-Plaza and Van Der Meulen 2001).

- Growth factors: diffusion of growth factors $D_{g}=0.045 \mathrm{~mm}^{2}$.day ${ }^{-1}$ was derived from in vitro TGF- $\beta$ and BMP molecular weight defintion (Joyce et al. 1990) related mathematically to the diffusion coefficient (Vander, Sherman, and Luciano 1998). A similar value of $0.0415 \mathrm{~mm}^{2}$.day ${ }^{-1}$ was estimated by BaílonPlaza et al. (Bailón-Plaza and Van Der Meulen 2001). In absence of data from experimental work, growth factors production rate coefficient $E_{g}=0.057$ ng. $\mathrm{ml}^{-1}$. (cells. $\left.\mathrm{ml}^{-1}\right)^{-1}$. day ${ }^{-1}$ was chosen according to Amor et al. model (Amor et al. 2011). Growth factors decay rate $d_{g}=100$ day $^{-1}$ was estimated from BaílonPlaza et al., using a growth factors half-life time of ten minutes (Bailón-Plaza and Van Der Meulen 2001; Coffey, Russell, and Barnard 1990).

- Bone formation: on the one hand, woven bone secretion by osteoblasts was modeled such as the mechanical influence compared to growth factors one is neglected for low mechanical strain. With higher mechanical stimuli, woven bone secretion is defined as a function of $g$, with $\alpha_{w}=2.5 \times 10^{-4}$ g.day ${ }^{-1}$ calibrated through a sensitivity analysis and compared with in vivo data (Abrahamsson et al. 2004; Berglundh et al. 2003). $\alpha_{w}$ and $\beta_{w}=10 \mathrm{ng} \cdot \mathrm{ml}^{-1}$ were chosen chosen with the same order of values of Baílon-Plaza et al. model (Bailón-Plaza and Van Der Meulen 2001). On the other hand, it is considered that woven bone remodeling into lamellar is mainly driven by mechanical factors (Davies 2003). A lamellar bone remodeling rate $\mathrm{Q}_{l_{0}}=0.9 \times 10^{-6} \mathrm{~mm}^{3}$.(g.day) ${ }^{-1}$ was obtained through a sensitivity analysis as well and compared with in vivo studies from scientific literature (Abrahamsson et al. 2004; Berglundh et al. 2003).

- Maximum cells, growth factors concentration and ECM density: It was estimated by Baílon-Plaza et al. that a typical concentration of cell involved in bone healing was $10^{6}$ cells.ml ${ }^{-1}$. Such value has been later used by several other computational models (Ambard and Swider 2006; Geris et al. 2008). Growth 
factors concentrations on the other hand are in the order of value of $10^{-9} \mathrm{~mol} / \mathrm{l}$ (Joyce et al. 1990). A typical associated concentration is 100 ng.ml-1, taking into account a average molecular weight of $100 \mathrm{~kg} / \mathrm{mol}$. Finally, an estimation made by Olsen et al. (Olsen et al. 1997) indicates a typical ECM density of $0.1 \mathrm{~g} . \mathrm{ml}^{-1}$ inside the healed bone area. According to all the values aforementioned, corresponding reciprocal of maximum constants $\alpha=10^{-} 6\left(\text { cells.ml }^{-1}\right)^{-1}$, $\kappa=10\left(\text { g.ml }{ }^{-1}\right)^{-1}$ and $\gamma=10^{-2}\left(\mathrm{ng} \cdot \mathrm{ml}^{-1}\right)^{-1}$ were defined to respectively create cells, ECM and growth factors saturation functions in the present model. 